

\title{
Euler Computations of Hypersonic Flow with Strong Blowing
}

\author{
Nelson Carter * \\ Bram van Leer ${ }^{\dagger}$ \\ The University of Michigan \\ Department of Aerospace Engineering \\ Ann $\Lambda$ rbor, MI 48109-2146
}

\begin{abstract}
A validation study is presented for a numerical code which calculates a two-dimensional steady-state solution for inviscid hypersonic flows in the presence of strong surface blowing. A higher-orde: Godunov-type finite-volume approach is used to discretize the inviscid Euler equations. Interface values of the state quantities are reconstructed using a monotone interpolation technique suggested by Koren, based on Van Leer's kappa scheme. The interface fluxes are computed using Roc's upwind-biased flux-difference splitting technique. The time-differencing algorithms used are a locally implicit, linearized Gauss-Seidel iteration scheme and an explicit multi-stage scheme with optimized short-wave damping. The results of the numerical calculations are compared with analytical solutions obtained for strong blowing along a flat plate and a wedge with an inversesquare-root injection-velocity distribution.
\end{abstract}

\section{Introduction}

The recent revival of interest in lypersonic vehicles has renewed interest in the study of hypersonic flow. One subject of study is the use of surface blowing to influence an external hypersonic flow field. Understanding of the interaction between the injected gas and the highspeed outer flow is critical to applications of blowing in propulsion, surface cooling, and control-force generation. Experiments in hypersonic flows, however, are difficult to perform; thus a heavy reliance on computational predictions has resulted. In the absence of experimental results, the only recourse is to validate the computational codes on the basis of flow cases for which analytical solutions exist. This can help to distinguish between physical and numerical effects in cases where only numerical results are available.

A generic problem is that of hypersonic flow over a

\footnotetext{
* Graduate Rescarch Student, Member AIAA

tProfessor, Member AIAA
}

flat plate with distributed blowing off the surface. The displacement effect of the blowing causes a shock wave to form ahead of the blowing region. If the blowing is of sufficient strength, the boundary layer is blown off; this is called "strong blowing". It results in a viscous free shear layer separating an essentially inviscid rotational blown layer next to the wall from an inviscid shock layer extending from the shear layer to the shock (Figure 1). For sufficiently high Reynolds numbers, the free shear layer can be assumed to have negligible thickness and be regarded as a slip-stream. This fully inviscid limiting case was considered two decades ago by Cole and Aroesty [1], and by Wallace and Kemp [2]. Recently, Messiter and Matarrese [3] have obtained similarity solutions that take into account the viscous interaction for an inverse-square-root distribution of the injection velocity along a flat plate and along a thin wedge, in two dimensions.

The present paper is a validation study for an Euler code for hypersonic flow with surface blowing developed by the authors. Numerical solutions obtained with a discretization of the Euler equations are compared with analytical solutions for the inviscid strong-blowing case. The numerical method used is a finite-volume technique for finding steady solutions to the two-dimensional Euler equations with boundary conditions consistent with the assumptions made in the analytical work. Comparisons for blowing off a flat plate and a wedge are presented.

\section{Summary of Analytical Work}

In the inviscid case to be considered, the flow field over a flat plate or a wedge in the presence of strong blowing can be separated into two different layers:

1. The blown layer next to the wall, made up of the injected gas.

2. The shock layer between the blown layer and the shock, made up of free-stream gas that has passed through the shock. 


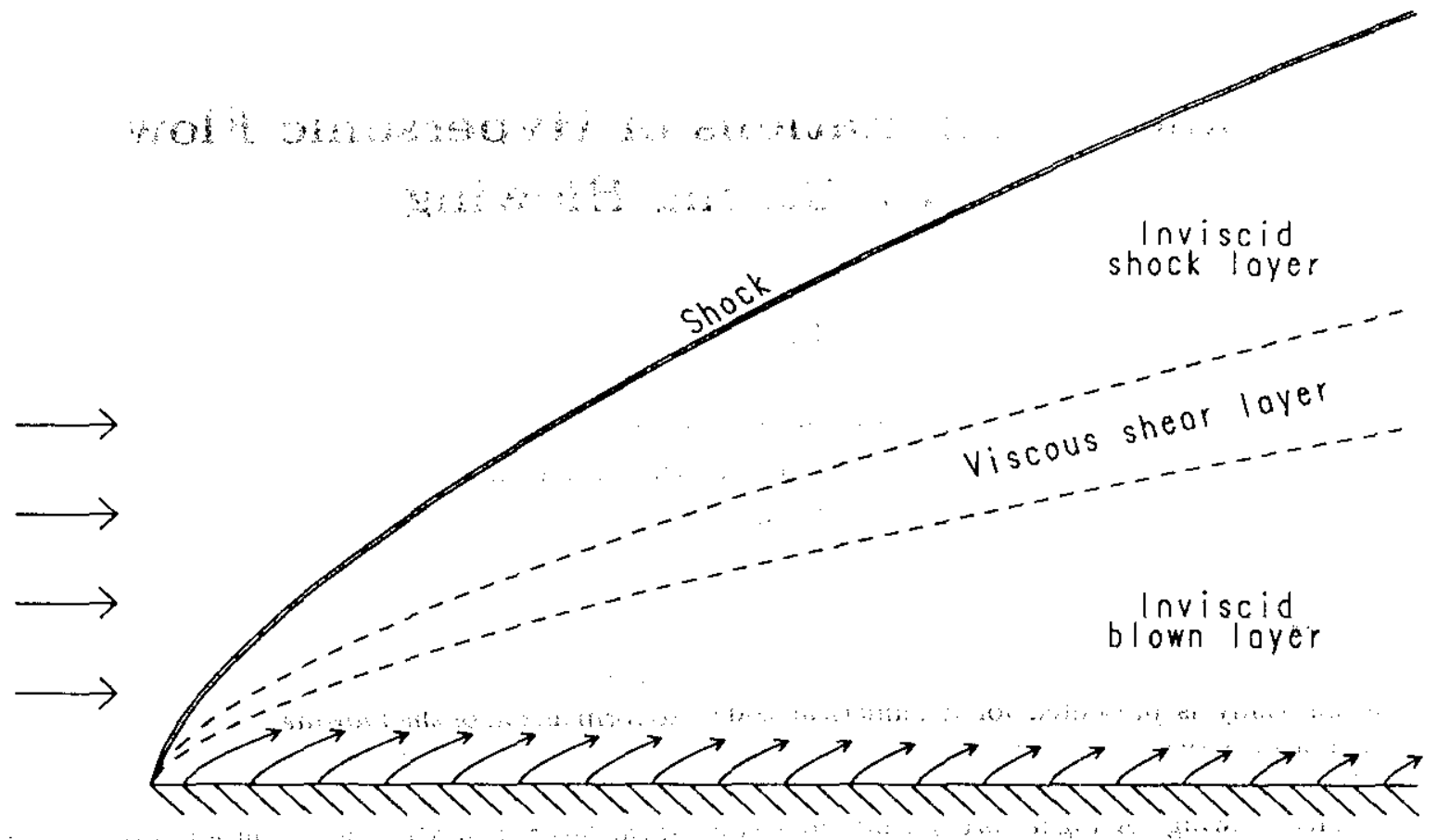

Figure 1: Schematic of the various layers present for strong blowing off a flat plate.

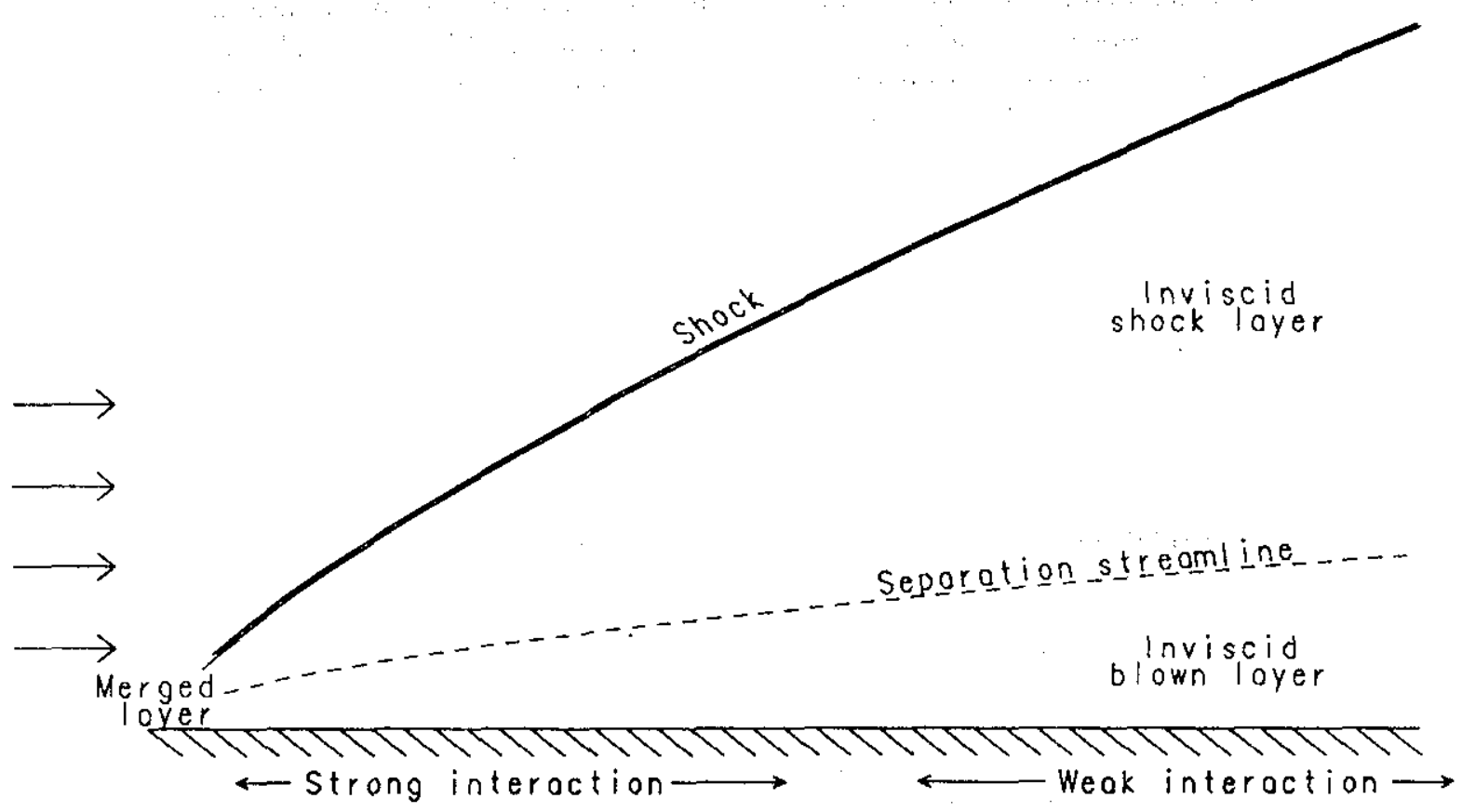

Figure 2: Schematic showing the inviscid case. The inviscid blown layer and the inviscid shock layer meet in a slip-stream along the separation streamline. 
The two layers meet in a slip-stream along the separating streamline (Figure 2). Flow in these layers can be characterized by three different regions along the plate or wedge. Far enough downstream, the relative pressure changes are small, and the interaction between the blown layer and the shock is described as weak (weak-interaction region). Further upstream, the curvature of the shock increases, and the relative pressure changes become large; here the interaction between the blown layer and shock is described as strong (strong-interaction region). Still further upstream, the thickness of the shock layer, blown layer, and free shear layer (whose thickness has been considered negligible up to this point) all become of the same order and no real distinction between them can be made. This region is referred to as the merged-layer regime. Similar flow classifications have been defined for viscous hypersonic flow in the absence of blowing and are discussed in books by IIayes and Probstein [4] and by Stewartson [5].

In the strong-interaction region, the flow in the shock layer is described by hypersonic small-disturbance theory. The flow in the blown layer is compressible, and is described by the so-called "inviscid boundary-layer equations". For flow on a flat plate, the results of Cole and Aroesty [1] and Messiter and Matarrese [3] may be used to show that an inverse square-root injection velocity distribution of the form

$$
\frac{v_{w}}{U_{\infty}}=0.3022 \frac{\delta^{2}}{\sqrt{\tilde{\rho}_{1 w}}} x^{-\frac{1}{2}}
$$

for $\gamma=1.4$, results in a separating streamline of the shape

$$
y_{s s}=\delta x^{\frac{3}{4}} ;
$$

the corresponding pressure distribution along the plate is

$$
\frac{p_{w}}{p_{\infty}}=1.119 M_{\infty}^{2} \delta^{2} x^{-\frac{1}{2}} .
$$

Here $\delta<<1$ is the blown-layer thickness at $x=1$, and $\tilde{\rho}_{1 w}$ is the ratio of the blown-gas density to the freestream density, assumed to be constant with a magnitude of $O(1)$. The coordinates $x$ and $y$ and thickness $\delta$ are non-dimensionalized with respect to the length of the plate. The strong-interaction results are appropriate for regions in which the parameter $M_{\infty}^{2} \delta^{2}$ is large $(\rightarrow \infty)$.

In the weak-interaction region, pressure changes are small and linearized supersonic flow theory can be applied. The pressure perturbations $\left(\frac{p-p_{\infty}}{p_{\infty}}\right)$ are proportional to the local slope of the effective body created by the blown layer $\left(y_{s s}^{\prime}\right)$. The flow inside the blown layer is assumed incompressible. Again, for a flat plate with an inverse square-root blowing distribution of the form

$$
\frac{v_{w}}{U_{\infty}}=\frac{\delta^{\frac{3}{2}}}{3.464}\left(M_{\infty} \tilde{\rho}_{1 w}\right)^{-\frac{1}{2}} x^{-\frac{1}{2}}
$$

[1] and [3] show that the resulting separating streamline has the form

$$
y_{s s}=\delta x^{\frac{2}{3}}
$$

the pressure distribution along the plate is

$$
\frac{p_{w}}{p_{\infty}}=1+\frac{2}{3} \gamma M_{\infty} \delta x^{-\frac{1}{3}} .
$$

These results are valid in regions where the parameters $y_{s}$ and $M_{\infty}$ are such that

$$
y_{s s}<<\frac{x}{M_{\infty}}
$$

i.e., the thickness of the blown layer $\left(y_{s s}\right)$ is small in comparison with the thickness of the shock layer, so that linearized supersonic flow theory is applicable.

These results have been extended in [1] and [3] to blowing along a slender wedge in hypersonic flow. In the strong-interaction region, the solution is the same as that for a flat plate since the wedge thickness is assumed to be small in comparison with the blown-layer thickness in this region. This requires that $y_{s s}>>x \alpha$, where $\alpha$ is the wedge half-angle.

The solution for the weak-interaction region is different for wedge flow. For the case of an inverse squareroot injection velocity distribution of the form

$$
\frac{v_{w}}{U_{\infty}}=\alpha^{2} \tilde{\rho}_{1 w}^{\frac{1}{2}}\left(\frac{\frac{\delta}{\alpha}}{1.7601}\right)^{\frac{3}{2}} x^{-\frac{1}{2}}
$$

the separating streamline retains its shape, i.e.,

$$
y_{s s}=\delta x^{\frac{2}{3}},
$$

but the pressure along the wedge becomes

$$
\frac{p_{w}}{P_{\infty}}=M_{\infty}^{2} \alpha^{2}\left(\frac{\gamma(\gamma+1)}{2}+2.0541 \frac{\delta}{\alpha} x^{-\frac{1}{3}}+\ldots\right) .
$$

The key parameter in this region is the ratio of the blown-layer thickness $\delta$ to the wedge half-angle $\alpha$. This ratio is assumed to be small. Also, as for the flat plate, the thickness of the blown layer is assumed to be small in comparison with the thickness of the shock layer.

\section{Numerical Strategy}

A higher-order Godunov-type [6] finite-volume approach was used to discretize the inviscid Euler equations. This requires a spacial interpolation routine, a numerical flux function, and a time-marching technique.

- State quantities in cell centers were interpolated at cell interfaces using a formula introduced by Van Leer [7][8]:

$$
\begin{aligned}
q_{i+\frac{1}{2}, j}=q_{i, j} & +\frac{1+\kappa}{4}\left(q_{i+1, j}-q_{i, j}\right) \\
& +\frac{1-\kappa}{4}\left(q_{i, j}-q_{i-1, j}\right) .
\end{aligned}
$$


This type of interpolation is at most second order accurate (for $\kappa=\frac{1}{3}$ ), and may lead to spurious oscilations in the solution in the neighborhood of flow discontinuities. To avoid this while maintaining the high order of accuracy in smooth flow regions, a limiter may be employed (Sweby [9]); the limiter adopted was one introduced by Koren [10] which is consistent with $\kappa=\frac{1}{3}$. It has the form

$$
\begin{gathered}
q_{i+\frac{1}{2}, j}=q_{i, j}+\frac{1}{2} \frac{2 R^{2}+R}{2 R^{2}-R+2}\left(q_{i, j}-q_{i-1, j}\right), \\
R=\frac{\left(q_{i+1, j}-q_{i, j}\right)}{\left(q_{i, j}-q_{i-1, j}\right)} .
\end{gathered}
$$

In order to apply Koren's interpolation formula uniformly, state quantities at the boundary were calculated first using an appropriate boundary proceedure, as explained below. The finite differences across the boundary were then replaced by twice the finite difference from the cell center to the boundary.

- Roe's approximate Riemann solver [11] was used to calculate the flux across each cell interface.

- Two different marching procedures were employed to reach the steady state solution.

1. On scalar machines, a locally implicit, linearized Gauss-Seidel iteration scheme was used. In order to avoid non-physical states (i.e. negative pressure) in the evolving flow, the time-step used was based on the ratio of the magnitude of the state vector to the magnitude of its rate of change. This is the Switched Evolution/Relaxation approach of Van Leer and Mulder [12].

2. On vector machines it appeared advantageous to use an explicit time-marching scheme. Multi-stage schemes developed by Van Leer, Tai, and Powell [13] with optimized shortwave damping vectorize well and are effective in avoiding non-physical transicnt states.

In order to make accurate comparisons with the analytical solutions, it is crucial to implement numerical boundary conditions that are consistent with the analytical ones (Figure 3).

- Along the inflow boundary of the grid, the flow normal to the boundary is supersonic inward, so there are no out-going characteristics. Thus, the flux into the cells along this boundary can be specified explicitly according to the free-stream state.

- Depending upon the shape of the grid chosen, the flux normal to the top boundary may be either supersonic or subsonic inward. If the flow is subsonic inward, disturbances from inside the computational domain must be allowed to propagate outward through the boundary. Along this upper boundary the flow is nearly uniform, so that the entropy is locally constant and derivatives along the boundary can be neglected. In a coordinate frame normal to the boundary one can define the Riemann invariants

$$
R^{ \pm}=u_{\perp} \pm \frac{2 a}{\gamma-1}
$$

transported normal to the boundary along characteristics with speed

$$
u_{\perp} \pm a
$$

Here $u_{\perp}$ is the component of the flow velocity normal to the boundary, and a is the local sound speed. Since the flow is subsonic inward, information from the interior propagates out along the $u_{1}+a$ characteristic. Thus the value of the $R^{+}$ Riemann invariant should be extrapolated toward the boundary in a manner consistent with the order of the spacial discretization. The inward flux is then completely determined by the $R^{+}$Ricmann invariant and three other flow quantities specified on the boundary according to the free-stream state.

- Along the boundary where the flow exits the grid, both supersonic and subsonic outflow exists. For the supersonic exit flow, no information can propagate in from outside the computional domain, thus the flux across the boundary is completely determined by the state quantities in the interior. For boundary cells where the flow is subsonic outward, information from outside can propagate in along the $u_{\perp}-a$ characteristic. Thus, some value of the $R^{-}$Riemann invariant should be specified; the problem is that the exterior flow conditions are not known here. Values from a known exact solution could be specified, but this would be incorrect when considering arbitrary blowing distributions. Hedstrom [14] proposed the time-dependent nonreflecting characteristic boundary condition

$$
\frac{\partial p}{\partial t}-\rho a \frac{\partial u_{\perp}}{\partial t}=0
$$

This type of boundary condition has the disadvantage that it produces a steady state dependent upon the initial conditions. An order-of-magnitude analysis of the governing equations shows that the pressure is constant across the blown layer; this condition can be enforced at the exit by using Hedstrom's boundary condition with the pressure gradient as a source term. The exit boundary condition then becomes

$$
\frac{\partial p}{\partial t}-\rho a \frac{\partial u_{\perp}}{\partial t}=-K\left(u_{\perp}-a\right) \frac{\partial p}{\partial y}, K>0 .
$$

This enforces the correct behavior of the pressure along the subsonic part of the outflow boundary. 


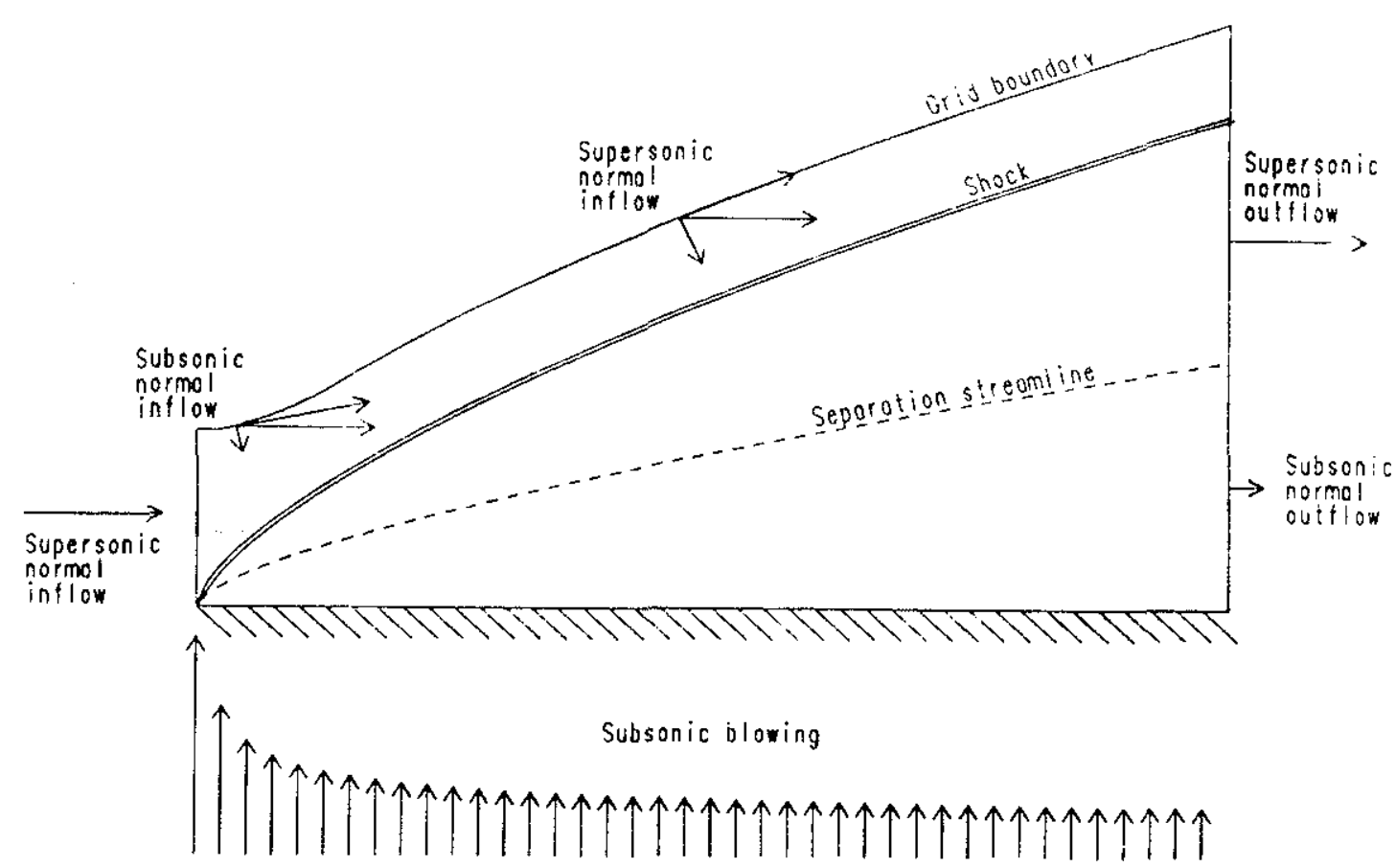

Figure 3: Schematic indicating the various types of boundary conditions encountered.

Typically values of $K$ between 0.1 and 1.0 were used. If $K$ is taken too small or too large, convergence to a steady state is delayed.

- Along the plate or wedge, the injected flow is subsonic inward, except very close to the nose where the flow can become supersonic for an $x^{-\frac{1}{2}}$ blowing distribution. Thus one can specify the normal velocity $v_{w}$, the tangential velocity (equal to zero for the cases considered), and the ratio of the injection density to the free-stream density $\tilde{\rho}_{1 w}$ (assumed constant along the wall). The wall pressure can then be determined by the $R^{-}$Riemann invariant extrapolated from the interior back toward the wall with the proper order of accuracy. Near the wall, the flow may not be locally isentropic; therefore, the approximate incremental form of the Riemann invariant.

$$
\Delta p-\ddot{\rho} a ̈ . \Delta u_{\perp}
$$

should be used for extrapolation; barred quantities refer to quantities averaged across the cell interfaces. The pressure then follows from

$$
\begin{gathered}
p_{w}-p_{i, j}-\rho_{i, j} a_{i, j}\left(u_{\perp w}-u_{\perp i, j}\right)= \\
\frac{1}{2}\left(\Delta p-\bar{\rho} \bar{a} \Delta u_{\perp}\right)_{\text {extrap }}
\end{gathered}
$$

The analytical solutions were employed in generating grids that would adequately resolve regions of interest in the flow field. Using the analytical predictions, grid points were clustered about the wall, the free shear layer and the shock (Figure 4). The clustering was based on an exponential spacing in the $y$ direction, and an algebraic spacing in the $x$ direction. Once a converged solution was obtained, this adaptive procedure was iterated on to achieve an optimal grid-point distribution for the case considered.

\section{Numerical Experiments}

Numerical experiments were conducted to obtain finitevolume solutions of the Euler equations for comparison with the analytical predictions for three different cases:

- Strong interaction on a flat plate.

- Predominantly weak interaction on a flat plate

- Predominantly weak interaction on a slender wedge

The case of strong interaction on a wedge was not considered since the theory assumes that the wedge thickness is small in comparison to the blown layer thickness. Thus to lowest order, the solution for strong interaction on a flat plate is recovered.

For the case of strong interaction on a flat plate. good agrcement has been obtained. The parameters chosen for this case are;

$$
\begin{gathered}
\delta=0.09, \\
M_{\infty}=20, \\
M_{\infty}^{2} \delta^{2}=3.24, \\
\tilde{\rho}_{1 w}=\frac{\rho_{\text {injected }}}{\rho_{\text {jree-stream }}}=1 .
\end{gathered}
$$

Early results on a rectangular grid showed poor agreement with the analytical solution. Cross-sectional profiles of the flow varibles indicated the presence of a viscous-like shear layer where the numerical code was 


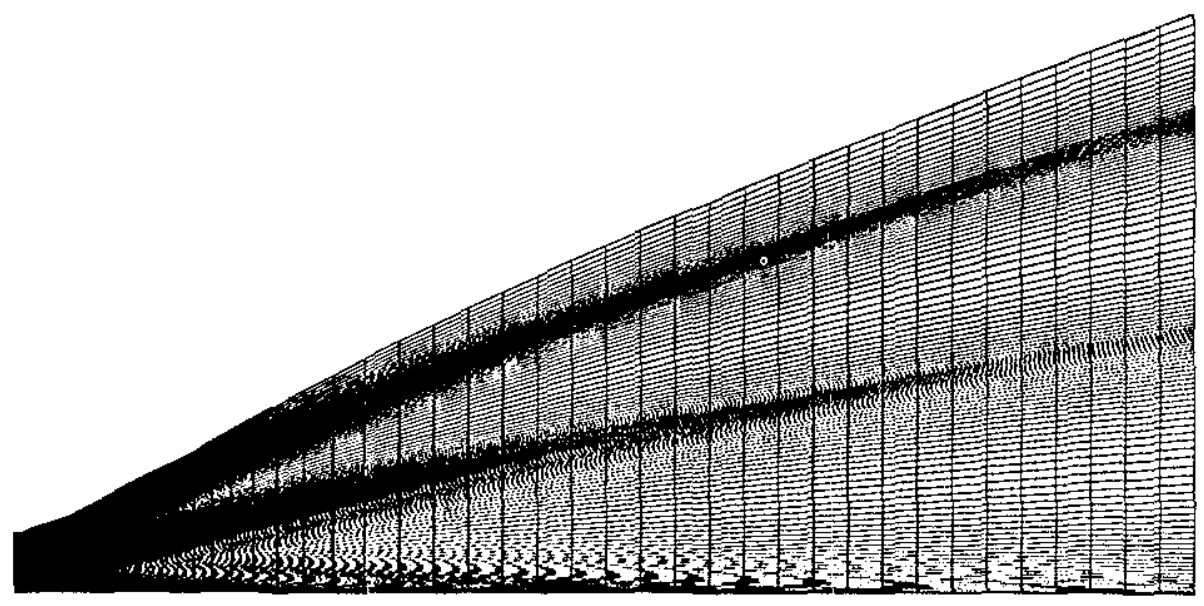

Figure 4: Computational grid.

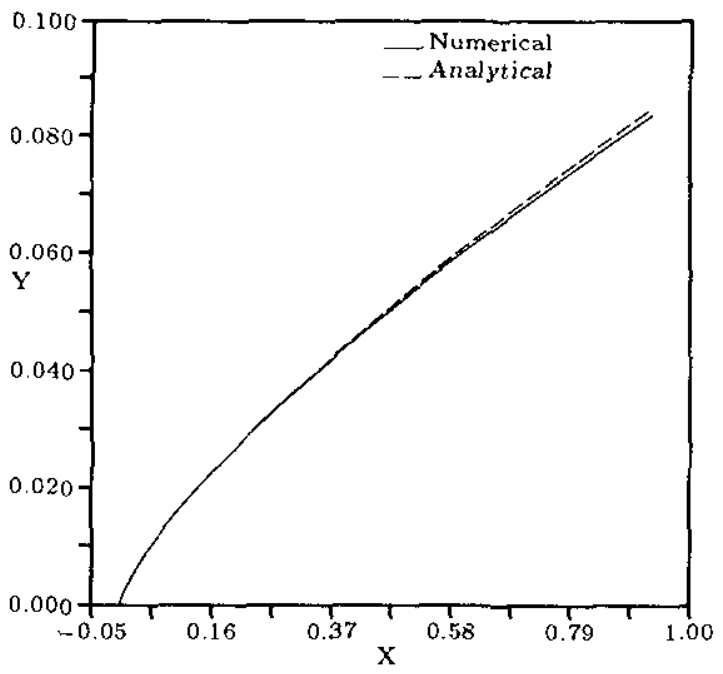

Figure 5: Seperating streamline shapes for strong interaction on a flat plate.

trying to model the free slip-stream. This is due to the artificial dissipation associated with the numerical scheme. Restructuring the grid to better fit the flow field, and clustering grid points near the numerical shear layer resulted in a reduction in the thickness of this layer. As the thickness of the layer decreased, the numerical solution approached the analytical solution.

A converged second-order solution on a $(83 \times 91)$ grid shows good agreement between the numerically determined blown-layer shape and the analytical prediction (Figure 5). The pressure distributions along the plate

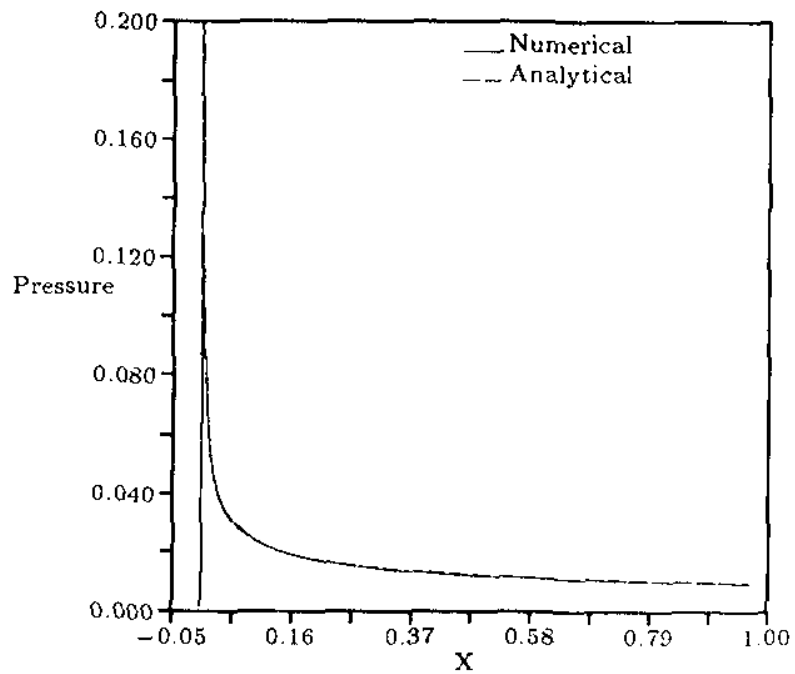

Figure 6: Strong interaction pressure distribution along a flat plate. 


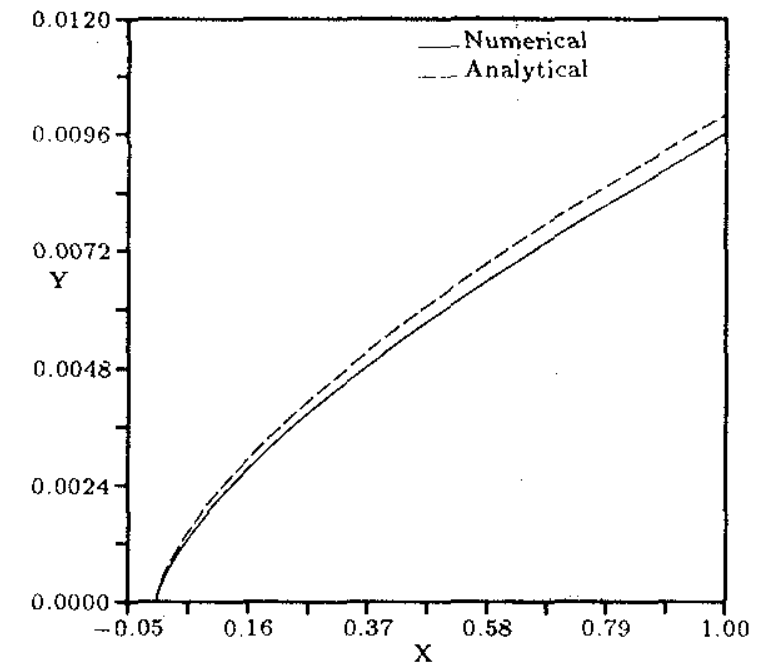

Figure 7: Separating streamline shapes for weak interaction on a flat plate.

also compare well with each other (Figure 6). Further grid refinements were attempted to verify that the numerical solution continued to approach the analytical solution as the mesh spacing decreased. It was found, however, that by refining the grid too far, the numerical dissipation can become so small that the solution bccomes unstable and exhibits features similar to those of a physically unstable shear layer (Figure 12).

Fair agreement with the analytical solution was obtained for the case of weak interaction on a flat plate. In this case, the blown layer thickness is thin in comparison with the shock layer. The shock is very weak, and lies roughly along the Mach lines. This case presents two difficultics. The magnitude of the blowing is much smaller so high resolution of the singular blowing distribution and flow features at the nose is even more important. Also, the thinness of the blown layer makes the solution more sensitive to the existence of a numerical shear layer whose thickness is grid dependent. The parameters chosen for this case are;

$$
\begin{gathered}
\delta=0.01, \\
M_{\infty}=10, \\
\tilde{\rho}_{1 w}=1 .
\end{gathered}
$$

A comparison between the analytical and the computational separating streamline shapes shows shows a discrepency of about five percent in the thickness of the blown layer (Figure 7). This discrepancy is also apparent in the pressure profiles along the plate (Figure 8).

The last case considered was that of weak interaction on a wedgc. For this case the parameters were chosen

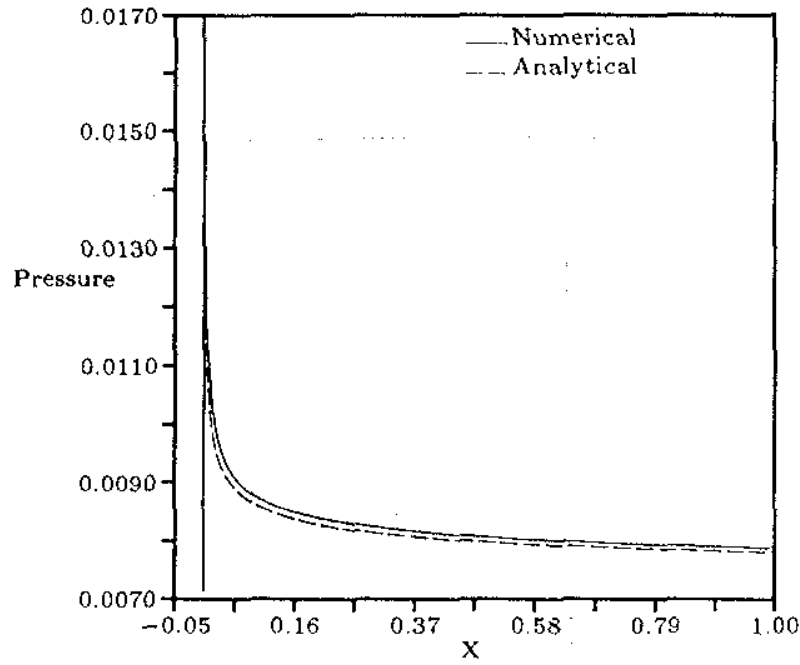

Figure 8: Weak interaction pressure distribution along a flat platc.

to be;

$$
\begin{gathered}
\frac{\delta}{\alpha}=0.1, \\
\alpha=8^{\circ}, \\
M_{\infty}=20, \\
\tilde{\rho}_{1 w}=1 .
\end{gathered}
$$

Figure 9 shows good agreement between the analytical and numerical separation streamline shapes, but Figure 10 shows that the numerically calculated pressure is higher than that predicted analytically. The analytical work assumes an infinite value of $\left(M_{\infty} \alpha\right)^{2}$. If large but finite values of $M_{\infty} \alpha$ are considered in the analysis, the resulting analytical pressure distribution,

$$
\frac{p_{w}}{P_{\infty}}=M_{\infty}^{2} \alpha^{2}\left(1.946+2.084 \frac{\delta}{\alpha} x^{-\frac{1}{3}}+\ldots\right),
$$

compares well with the numerically determined values (Figure 11).

\section{Further Work}

With the validation study completed, the code will be used to obtain flow solutions in cases where no analytical predictions exist. Of particular interest is strip blowing as a practical means to control the pressure distribution along the surface. The code has been expanded to include the effects of physical viscosity and heat conduction, i.e. to approximate the Navier-Stokes equations. For validation of the code in viscous cases, analytical solutions are again avaiable [3]. A future paper will include some comparisons in the viscous regime. 


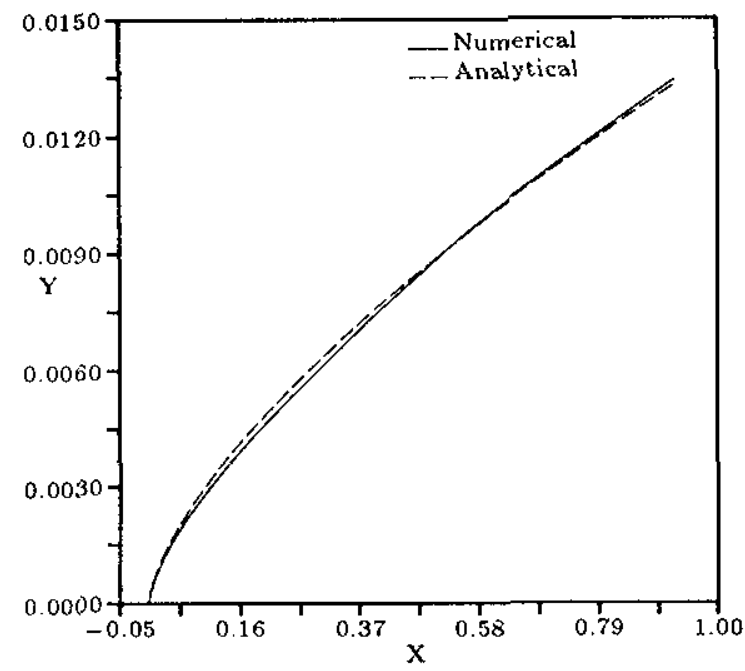

Figure 9: Separating streamline shapes for weak interaction on a wedge.

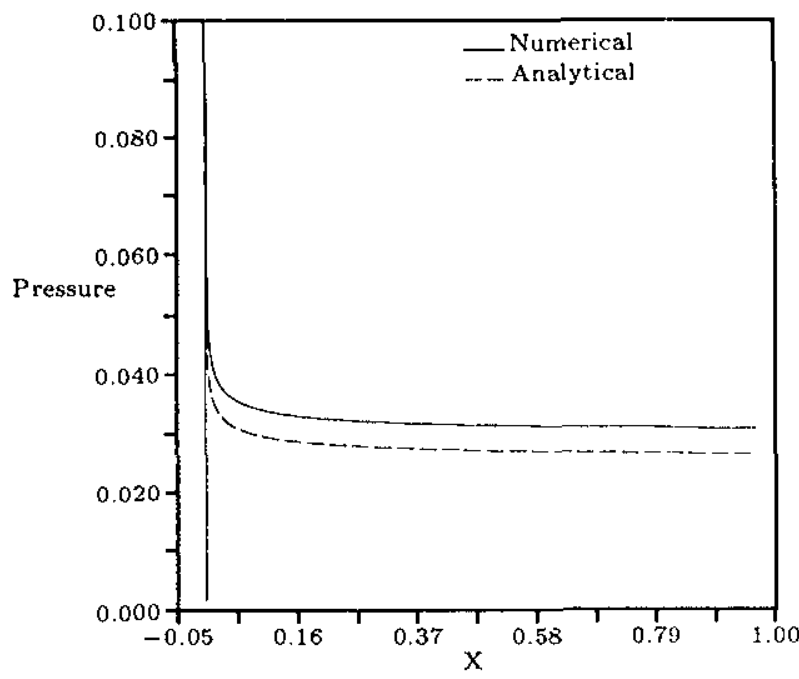

Figure 10: Weak interaction pressure distributions along a wedge.

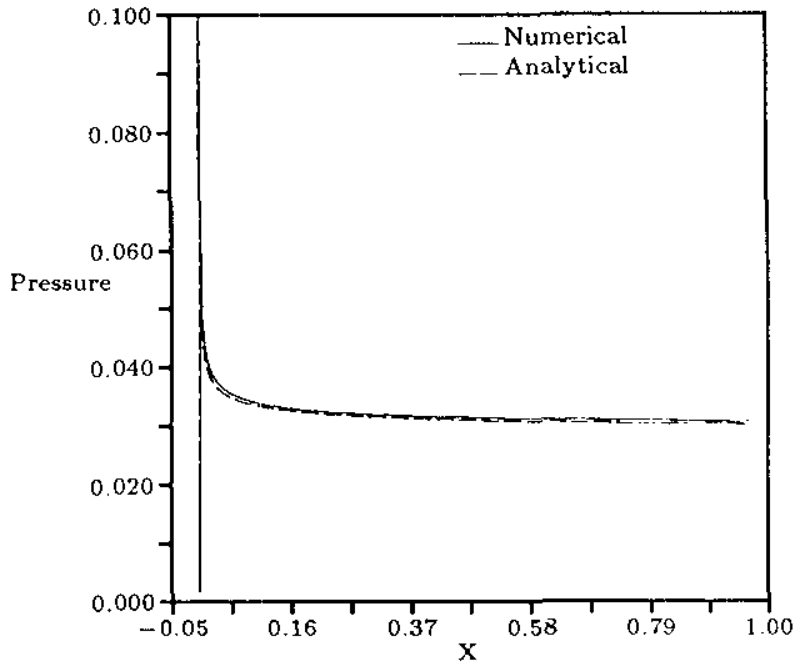

Figure 11: Weak interaction pressure distribution comparison with finite $M_{\infty} \alpha$ correction.

\section{Acknowledgement}

Supported in part by the U. S. Army Strategic Defence Command.

\section{References}

[1] J. D. Cole and J. Aroesty, "The blowhard probleminviscid flows with surface blowing," Journal of Heat and Mass Transfer, vol. 11, 1967.

[2] J. Wallace and N. Kemp, "Similarity solutions to the massive blowing problem," AIAA Journal, vol. 7, 1969.

[3] A. F. Messiter and M. Matarrese, "Iyypersonic viscous interaction with strong blowing," in preparation, 1989.

[4] W. D. Ilayes and R. F. Probstein, Iypersonic Flow Theory. Academic Press, 1959.

[5] K. Stewartson, The Theory of Laminar Boundary Layers in Compressible Fluids. Oxford University Press, 1964.

[6] B. van Leer, "Towards the ultimate conservative difference scheme. V. A second-order sequel to Godunov's method," Journal of Computational Physics, vol. 32, 1979

[7] B. van Leer, "Upwind-difference methods for aerodynamic problems governed by the Euler equations," in Large-Scale Computations in Fluid Mechanics, Lectures in Applied Mathematics, vol. 22, 1985 . 


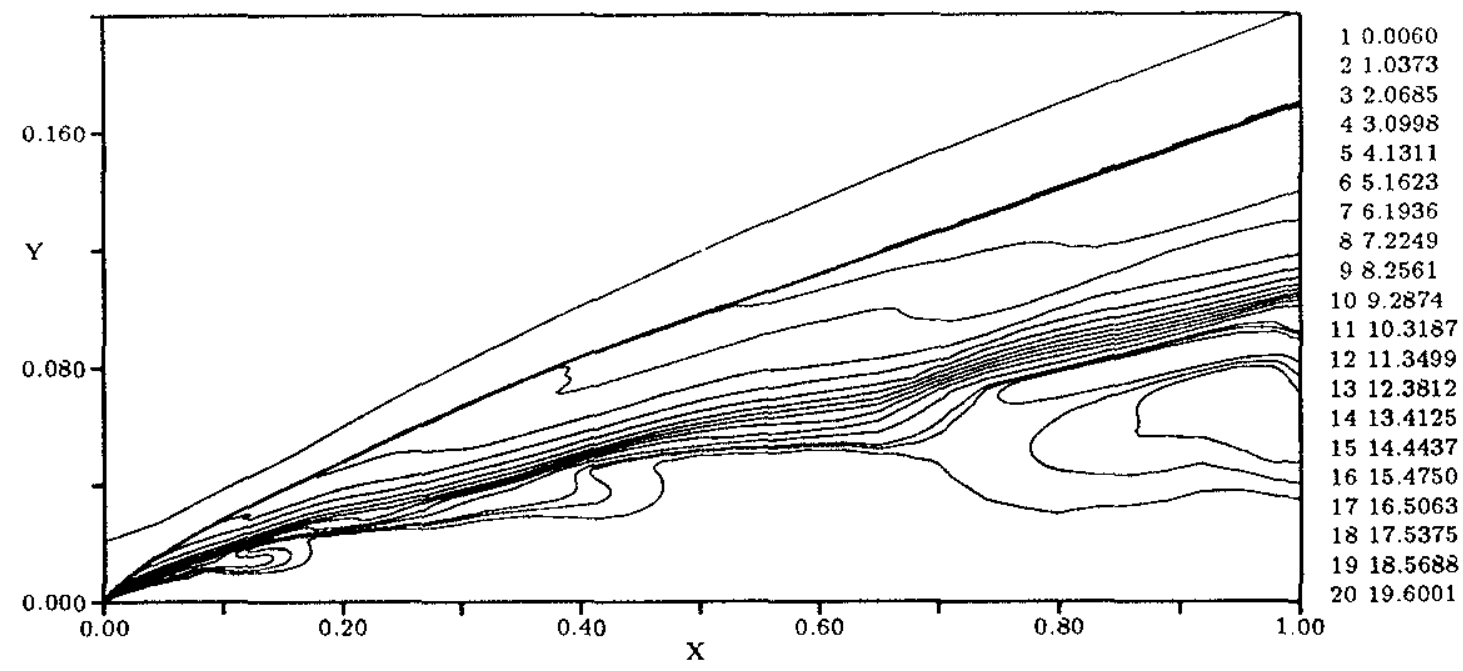

Figure 12: Example of an unstable shear layer encountered in a higher-order calculation on a refined grid.

[8] W. K. Anderson, J. L. Thomas, and B. van Leer, "Comparison of finite volume flux vector splittings for the Euler equations," AIAA Journal, vol. 24, no. $9,1986$.

[9] P. K. Sweby, "High resolution schemes using flux limiters for hyperbolic conservation laws," SIAM Journal on Numerical Analysis, vol. 21, 1984.

[10] B. Koren, Multigrid and Defect Correction for the Navier-Stokes equations. $\mathrm{PhD}$ thesis, Delft University, 1989.

[11] P. L. Roe, "Approximate Riemann solvers, parameter vectors, and difference schemes," Journal of Computational Physics, vol. 43, 1981.

[12] B. van Leer and W. A. Mulder, "Relaxation methods for hyperbolic equations," in Numerical Methods for the Euler Equations of Fluid Dynamics, 1985 .

[13] B. van Leer, C. H. Tai, and K. G. Powell, "Design of optimally-smoothing multi-stage schemes for the Euler equations," in AIAA glh Compulational Fluid Dynamics Conference, 1989.

[14] G. W. Hedstrom, "Nonreflecting boundary conditions for nonlinear hyperbolic systems," Journal of Computational Physics, vol. 30, 1970. 